Alvin Lee Day, MD

Clinical Assistant Professor, Division of

Rheumatology, Palmetto Health/University

of South Carolina School of Medicine,

Columbia, SC
Kevin L. Winthrop, MD, MPH

Professor, Division of Infectious Diseases,

Oregon Health and Science University,

Portland, OR
Jeffrey R. Curtis, MD, MS, MPH

Professor, Division of Clinical Immunology and Rheumatology, University of Alabama at Birmingham, Birmingham, AL

\title{
The effect of disease-modifying antirheumatic drugs on vaccine immunogenicity in adults
}

\section{ABSTRACT}

Patients with immunocompromising conditions are at higher risk of vaccine-preventable infections. Further, those receiving immunosuppressive disease-modifying antirheumatic drugs (DMARDs) can have variable responses to vaccines depending on which vaccine and which DMARD they are receiving.

\section{KEY POINTS}

Influenza vaccine should be given yearly to all patients on DMARDs, with modification to either the timing of DMARD or vaccine administration for patients receiving methotrexate or rituximab.

Pneumococcal vaccination should be given to all patients on DMARDs beginning at age 19 with pneumococcal 13-valent conjugate vaccine (PCV13) and then the 23-valent pneumococcal polysaccharide vaccine (PPSV23). Methotrexate, abatacept, tofacitinib, and rituximab reduce pneumococcal vaccine immunogenicity.

The live herpes zoster vaccine is contraindicated in those with severe immunosuppression (eg, those on biologics or Janus kinase inhibitors) but may be given to those on conventional synthetic DMARDs.

Limited data exist on the effects of DMARDs on human papillomavirus vaccine.

Dr. Winthrop has disclosed consulting for Abbvie Pharmaceuticals, Bristol-Myers Squibb, Eli Lilly, Galapagos, Genentech/Roche, Gilead Sciences, GlaxoSmithKline, Pfizer, Regeneron, Sanofi, and UCB. Dr. Curtis has disclosed consulting, speaking, or honoraria for Abbvie, Amgen, BMS, Corona, Gilead, Janssen, Lilly, Myriad, Novartis, Pfizer, Sanofi, and Scipher.

Dr. Day reports no relevant financial relationships which, in the context of his contribution, could be perceived as a potential conflict of interest.

doi:10.3949/ccjm.87a.20056
D ATIENTS CAN BECOME immunocompromised from primary or secondary causes. Primary causes are typically inherited, whereas secondary causes may be iatrogenic (ie, medication-related) or due to the underlying disease process. Infections represent a serious risk to patients who are immunocompromised, and the US Centers for Disease Control and Prevention (CDC) has developed specific vaccination recommendations for these individuals beginning at age $19 .{ }^{1}$

Live vaccines are contraindicated in the severely immunocompromised, which, in patients receiving immunosuppressive drugs, is defined as those receiving any of the following:

- Prednisone in a dosage of $2 \mathrm{mg} / \mathrm{kg}$ or more, or more than $20 \mathrm{mg} /$ day

- Methotrexate in a dosage of more than 0.4 $\mathrm{mg} / \mathrm{kg} /$ week

- Azathioprine more than $3 \mathrm{mg} / \mathrm{kg} /$ day

- 6-Mercaptopurine more than $1.5 \mathrm{mg} / \mathrm{kg} /$ day

- Any biologic agent. ${ }^{2}$

In this review, we discuss the use of various vaccines in immunocompromised patients, with a focus on iatrogenic immunosuppression for patients with systemic rheumatic or other immune-mediated inflammatory diseases.

\section{IMMUNE-MEDIATED INFLAMMATORY DISEASES AND INFECTION}

Patients with immune-mediated inflammatory diseases such as rheumatoid arthritis, psoriatic arthritis, and Crohn disease are at increased risk of infections, often due to the immunosuppressive medications they need (Table 1). 


\section{TABLE 1}

\section{Selected disease-modifying antirheumatic drugs \\ Antimetabolite \\ Methotrexate \\ Tumor necrosis factor inhibitors \\ Adalimumab \\ Certolizumab \\ Etanercept \\ Golimumab \\ Infliximab}

Anti-CD80/CD86

Abatacept

\section{Janus kinase inhibitors \\ Baricitinib \\ Tofacitinib \\ Upadacitinib}

Anti-CD20

Rituximab

Interleukin (IL-) 6 inhibitors

Sarilumab

Siltuximab

Tocilizumab

\section{IL-17 inhibitors}

Brodalumab

Ixekizumab

Secukinumab

should be

offered when

appropriate

to reduce risk

\section{IL-12/23 inhibitors}

Ustekinumab

A large, retrospective US study ${ }^{3}$ evaluated the incidence of hospitalization for infections in patients with rheumatoid arthritis who had no exposure to a biologic agent in the year preceding the study compared with those who switched among various biologic agents in the year preceding the study. The mean rate of hospitalization for infections was 4.6 per 100 person-years in biologic-naive patients, compared with 7.0 for biologic-experienced patients switching to a new therapy. This suggests that those with more refractory disease (using switching of biologic drugs as a proxy for more treatment-refractory disease) were at greater risk of infection. Pneumonia and softtissue infections were the most common types of infections.

Risk stratification for patients at high risk is important in both counseling patients and addressing modifiable risk factors for infection (eg, vaccination, tobacco use, glucocorticoid use). Infection risk calculators, such as the Rheumatoid Arthritis Observation of Biologic Therapy (RABBIT) Risk Score ${ }^{4}$ or similar approaches developed for use in large administrative databases, ${ }^{5}$ have been developed to estimate the yearly probability of a serious infection. The risk of most if not all types of infections is increased in patients with immunemediated inflammatory diseases, and certain therapies for these disease further increase the risk. For example, the incidence of herpes zoster is higher in immune-mediated inflammatory diseases than in the general population and is further increased with Janus kinase inhibitors. ${ }^{6}$

More broadly, a systematic literature review of articles published from October 2009 to August 2018 was performed to determine the incidence and prevalence of vaccinepreventable illnesses in patients with autoimmune inflammatory rheumatic diseases. ${ }^{7}$ Of the 3,876 articles initially retrieved, 63 met the inclusion criteria that allowed for analysis of incidence and prevalence rates of influenza, pneumococcal disease, hepatitis $\mathrm{B}$, herpes zoster, and human papillomavirus (HPV) infection. The rates of influenza, Pneumococcus, herpes zoster, and HPV infections were higher than those in the general population.

Due to the significant risk of infection in patients with autoimmune inflammatory rheumatic diseases, vaccines should be offered when appropriate to reduce the risk. ${ }^{1,8}$

\section{INFLUENZA VACCINATION}

All adults, regardless of immunocompromised status, should receive a single dose of the annual influenza vaccine each year. Immunocompromised patients should receive either the recombinant influenza vaccine or the inactivated influenza vaccine ${ }^{1}$; the live attenuated influenza vaccine is contraindicated in this population. An egg allergy is not an absolute contraindication, as cell-culture based vaccines are available. ${ }^{9}$

\section{Which influenza vaccine to use?}

The standard inactivated influenza vaccine is trivalent, containing 2 influenza A strains and 1 influenza B strain. A quadrivalent vaccine, 
TABLE 2

\section{Impact of disease-modifying antirheumatic drugs on vaccine immunogenicity}

\begin{tabular}{|c|c|c|c|c|c|c|c|}
\hline DMARDs & $\begin{array}{l}\text { Influenza } \\
\text { vaccine }\end{array}$ & PPSV23 & PCV7/13 & $\begin{array}{l}\text { Live zoster } \\
\text { vaccine }\end{array}$ & $\begin{array}{l}\text { Recom- } \\
\text { binant } \\
\text { zoster } \\
\text { vaccine }\end{array}$ & $\begin{array}{l}\text { Hepatitis B } \\
\text { vaccine }\end{array}$ & $\begin{array}{l}\text { Human } \\
\text { papilloma- } \\
\text { virus } \\
\text { vaccine }\end{array}$ \\
\hline Methotrexate & Decrease $e^{14,15}$ & Decrease $e^{14,24}$ & Decrease $^{28}$ & No effect ${ }^{17,34}$ & Not studied & Not studied & No effect ${ }^{40,41}$ \\
\hline TNF inhibitors & No effect ${ }^{14}$ & No effect ${ }^{14,24}$ & No effect ${ }^{28,29}$ & $\begin{array}{l}\text { Study pending, } \\
\text { contraindicated }\end{array}$ & $\begin{array}{l}\text { Study } \\
\text { pending }\end{array}$ & Decrease $\mathrm{e}^{37-39}$ & No effect ${ }^{40,41}$ \\
\hline Abatacept & No effect ${ }^{16}$ & No effect ${ }^{16}$ & Decrease $^{30}$ & $\begin{array}{l}\text { Study pending, } \\
\text { contraindicated }\end{array}$ & $\begin{array}{l}\text { Study } \\
\text { pending }\end{array}$ & Not studied & Not studied \\
\hline $\begin{array}{l}\text { Janus kinase } \\
\text { inhibitors }\end{array}$ & No effect ${ }^{17}$ & Decrease $^{17}$ & No effect ${ }^{31,32}$ & $\begin{array}{l}\text { Not studied, } \\
\text { contraindicated }\end{array}$ & $\begin{array}{l}\text { Study } \\
\text { pending }\end{array}$ & Not studied & Not studied \\
\hline Rituximab & $\begin{array}{l}\text { De- } \\
\text { crease }^{14,18,19}\end{array}$ & Decrease ${ }^{19,25}$ & Decrease $\mathrm{e}^{30,33}$ & $\begin{array}{l}\text { Not studied, } \\
\text { contraindicated }\end{array}$ & No effect ${ }^{36}$ & Not studied & Not studied \\
\hline IL-6 inhibitors & No effect ${ }^{20}$ & No effect ${ }^{20}$ & No effect ${ }^{30}$ & $\begin{array}{l}\text { Not studied, } \\
\text { contraindicated }\end{array}$ & Not studied & Not studied & Not studied \\
\hline IL-17 inhibitors & No effect ${ }^{21-23}$ & No effect ${ }^{26}$ & Not studied & $\begin{array}{l}\text { Not studied, } \\
\text { contraindicated }\end{array}$ & Not studied & Not studied & Not studied \\
\hline $\begin{array}{l}\text { IL-12/23 } \\
\text { inhibitors }\end{array}$ & Not studied & No effect ${ }^{27}$ & Not studied & $\begin{array}{l}\text { Not studied, } \\
\text { contraindicated }\end{array}$ & Not studied & Decrease $^{38}$ & Not studied \\
\hline
\end{tabular}

DMARDs = disease-modifying antirheumatic drugs; IL = interleukin; PCV7/13 = 7- or 13-valent pneumococcal conjugate vaccine; PPSV23 = 23-valent pneumococcal polysaccharide vaccine; TNF = tumor necrosis factor

also available, contains the standard strains with an additional influenza B (Yamagata) strain. A high-dose trivalent vaccine can be considered in individuals over age 65 , as it confers a higher percentage of protective titers than the standard-dose vaccine and has been shown to have greater clinical effectiveness in preventing influenza infection. ${ }^{10}$

The recommendation to use the high-dose vaccine in at-risk individuals was further supported by a 2019 trial from Hong Kong that enrolled community-dwelling adults ages 65 to $82 .{ }^{11}$ Sera were collected before and after vaccination with the 2017-2018 standarddose quadrivalent, the trivalent with MF59 adjuvant, the high-dose trivalent, or the recombinant hemagglutinin quadrivalent vaccine. The MF59-adjuvanted trivalent, high-dose trivalent, and recombinant-hemagglutinin quadrivalent vaccines are considered enhanced vaccines, as either the increased dosage or use of an adjuvant causes a more robust immunogenic response. The mean rise in titer to egg-propagated $\mathrm{H} 1 \mathrm{~N} 1$ and $\mathrm{H} 3 \mathrm{~N} 2$ and microneutralized $\mathrm{H} 3 \mathrm{~N} 2$ was significantly higher in all enhanced-vaccine groups than in the group that received the standard-dose quadrivalent vaccine.

Enhanced vaccination in patients with immune-mediated inflammatory diseases was evaluated in a randomized controlled trial in patients with rheumatoid arthritis. ${ }^{12}$ The high-dose trivalent vaccine was compared with the standard-dose quadrivalent vaccine in 279 seropositive patients on conventional synthetic disease-modifying antirheumatic drugs (DMARDs), biologic DMARDs (tumor necrosis factor [TNF] inhibitors, anti-interleukin-6 [anti-IL-6]), or Janus kinase inhibitors. Even though this group of individuals was not selected for being age 65 or older (the mean age was $61.0 \pm 12.9$ years), the high-dose trivalent vaccine significantly improved immunogenicity compared with the standard- 
dose vaccine. While clinical outcomes (eg, incidence of influenza infection) were not assessed, this laboratory finding likely indicates that high-dose vaccination is preferable for all rheumatoid arthritis patients, irrespective of age.

The choice of influenza vaccine may also depend on local virulence patterns, as the Yamagata strain, which is not covered by the high-dose trivalent vaccine, may be the primary strain, or at least a relatively common strain. Although not as common on a national scale in recent years, the Yamagata strain varies in prevalence from year to year and has accounted for a significant portion of influenza $B$ in the recent past. A high-dose quadrivalent influenza vaccine that includes coverage for the Yamagata strain will be available for the 2020-2021 influenza season. ${ }^{13}$

\section{Effect of DMARDs}

\section{on influenza vaccine effectiveness}

Most DMARDs do not have a major effect on influenza vaccine seroprotection (Table 2)..$^{14-41}$ However, rituximab significantly reduces it. ${ }^{14,18,19}$ Rituximab is typically given every 6 months, and vaccination should be
A high-dose quadrivalent flu vaccine that covers the Yamagata strain will be available

for the 2020-2021 season given about 2 weeks before the next rituximab dose. ${ }^{18}$

Methotrexate also decreases seroprotection from the influenza vaccine, but to a lesser degree than rituximab. ${ }^{14,15}$ Holding methotrexate dosing for 2 weeks after influenza vaccination can improve vaccine seroprotection, as was demonstrated in a randomized controlled trial conducted among rheumatoid arthritis patients in Korea. ${ }^{15}$ The diminution of beneficial effect of vaccination was related to methotrexate dose, and patients receiving $15 \mathrm{mg}$ or more per week had a more reduced response than those on lower methotrexate doses. Patients on even lower but commonly used methotrexate doses had a minimal effect of methotrexate on vaccine immunogenicity.

TNF inhibitors, ${ }^{14}$ abatacept,${ }^{16}$ tofacitinib, ${ }^{17}$ tocilizumab, ${ }^{20}$ and secukinumab ${ }^{21-23}$ have not been shown to substantially reduce the proportion of patients who achieve adequate seroprotection.

While most studies have evaluated only the laboratory outcome of immunogenicity as a surrogate for clinical effectiveness, some observational studies have examined clinical outcomes such as the incidence of infection. ${ }^{42}$ A retrospective observational study ${ }^{42}$ of 30,788 patients with immune-mediated inflammatory diseases compared those who received and did not receive vaccination. In propensity score-adjusted analysis, vaccination reduced the risks of:

- Influenza-like illness (adjusted hazard ratio [aHR] 0.70, 95\% confidence interval [CI] 0.54-0.92)

- Hospitalization for pneumonia (aHR 0.61, 95\% CI 0.50-0.75)

- Hospitalization for chronic obstructive pulmonary disease exacerbation (aHR $0.67,95 \%$ CI 0.46-0.99)

- Death due to pneumonia (aHR 0.48, 95\% CI 0.33-0.71).

\section{PNEUMOCOCCAL VACCINATION}

For immunocompromised patients such as those with immune-mediated inflammatory diseases, pneumococcal vaccination is recommended starting at age 19. ${ }^{1}$ Immunocompromised individuals should first receive a single dose of PCV13. A dose of PPSV23 follows, at least 8 weeks later. A second dose of PPSV23 is recommended 5 years after the first dose of PPSV23. After a second dose of PPSV23, no further booster vaccinations are recommended. Additionally, individuals who received PPSV23 before age 65 for any indication should receive another dose at least 5 years later. For those who received PPSV23 before PCV13, PCV13 should be given at least 1 year after PPSV23.

\section{Effect of DMARDs on pneumococcal vaccine effectiveness}

Similar to influenza vaccination, most DMARDs have limited effects on pneumococcal vaccine immunogenicity (Table 2). Methotrexate and rituximab, however, decrease the humoral response to pneumococcal vaccine. ${ }^{14,19,24,25,28-30,33,36}$

A systematic review and meta-analysis was performed to determine the effects of methotrexate, TNF inhibitors, and rituximab on the immunogenicity of the influenza and pneumococcal vaccines in patients with rheumatoid arthritis. ${ }^{14}$ Twelve studies were included in the analysis, but only 2 of them specifically 
tested methotrexate's effect on pneumococcal vaccine effectiveness. ${ }^{24,28}$ Methotrexate significantly reduced the vaccine response against pneumococcal serotypes $6 \mathrm{~B}$ and $23 \mathrm{~F}$, with a pooled odds ratio (OR) of $0.33(95 \%$ CI $0.20-0.54)$ for $6 \mathrm{~B}$ and $0.58(0.36-0.94)$ for 23F. These serotypes were chosen because they were commonly seen in invasive pneumococcal disease both worldwide and in Sweden, where the study was performed.

Similarly, only 2 of the studies evaluated the effect of rituximab. ${ }^{19,33}$ Serotype $6 \mathrm{~B}$ immunogenicity was significantly reduced with rituximab (OR 0.25, 95\% CI 0.11-0.58), and there was a trend toward a similar reduction for serotype 23F (OR 0.21, 95\% CI 0.041.05). Later studies have also shown a significant reduction in both $6 \mathrm{~B}$ and $23 \mathrm{~F}$ serotype immunogenicity with rituximab compared with controls. ${ }^{30}$ The addition of methotrexate to rituximab further reduced immunogenicity.

Similar to the recommendation for the timing of influenza vaccination in patients treated with rituximab, pneumococcal vaccination should be given as close to the start of a subsequent rituximab dosing cycle as possible (eg, approximately 2 weeks before the next rituximab cycle).

Tofacitinib also decreases the humoral response to PPSV $23,{ }^{17}$ yet both tofacitinib and baricitinib showed that a high percentage of patients who received PCV13 while on these treatments were able to mount a satisfactory immune response, although there was no control group in those studies. ${ }^{31,32}$ TNF inhibitors have not been shown to have a significant effect on humoral response in PPSV23 $3^{14,24}$ or $\mathrm{PCV} 7{ }^{28}$ in the absence of concomitant methotrexate. Tocilizumab did not reduce response to PPSV2320 or PCV7. ${ }^{30}$ Ixekizumab $^{26}$ and ustekinumab ${ }^{27}$ did not significantly reduce immunogenicity to PPSV23 in healthy controls or in patients with moderate-to-severe psoriasis respectively, but PCV13 has not been studied for patients receiving these classes of biologics.

\section{HERPES ZOSTER VACCINATION}

Herpes zoster vaccination in the general population is recommended starting at age 50 with a 2 -dose series of recombinant zoster vaccine. ${ }^{1}$
Many primary care practices have stopped using the live zoster vaccine (Zostavax), as recombinant zoster vaccine (Shingrix) is more effective, ${ }^{43}$ and the live zoster vaccine was discontinued in the United States in July 2020.44

The guideline published by the American College of Rheumatology in 2015 recommended live zoster vaccination for all patients with rheumatoid arthritis who are at least age $50 .{ }^{8}$ Recommendations to use recombinant zoster vaccine among rheumatology patients have not yet been formulated or issued, and we currently have few data on its efficacy, safety (eg, risk of disease flare), and systemic reactogenicity in these populations.

Recombinant zoster vaccine is not a live vaccine. However, its clinical trials excluded people who were considered severely immunocompromised and also those with rheumatoid arthritis, systemic lupus erythematosus, and similar diseases receiving typical immunomodulatory therapies (eg, conventional synthetic DMARDs, biologics, and Janus kinase inhibitors). There is at least the potential concern for flare of underlying autoimmune conditions with recombinant zoster vaccine due to the potent immune response stimulated by the adjuvant. ${ }^{45}$ Recombinant zoster vaccine is currently being studied in patients with immune-mediated inflammatory diseases and a variety of other immunocompromised patient populations. ${ }^{1}$

Although recombinant zoster vaccine is not yet recommended for patients with immune-mediated inflammatory diseases, a retrospective review of 300 patients with rheumatic disease who received it showed only a 3\% incidence of rheumatoid arthritis flare within 12 weeks of vaccination and no cases of herpes zoster reactivation. ${ }^{46}$ Key limitations of this study included retrospective flare ascertainment, as recorded by documentation in rheumatologists' medical records, rather than prospective and systematic capture of flare and severe reactogenicity according to validated prespecified case definitions.

Despite US recommendations that favor recombinant over live zoster vaccine for healthy older patients, there are a number of countries worldwide in which it is not available, and the live vaccine remains the only option for herpes zoster vaccination. How- 
ever, since it is a live vaccine, there are potential concerns about transmitting infection to patients with severe immunosuppression. The $\mathrm{CDC}^{47}$ says its use is acceptable for patients treated with:

- Methotrexate $\leq 0.4 \mathrm{mg} / \mathrm{kg} /$ week

- Azathioprine $\leq 3.0 \mathrm{mg} / \mathrm{kg} / \mathrm{day}$

- 6-Mercaptopurine $\leq 1.5 \mathrm{mg} / \mathrm{kg} / \mathrm{day}$

- Prednisone $<20 \mathrm{mg} /$ day or equivalent

- Intra-articular, intrabursal, or peritendinous corticosteroid injections.

For patients with rheumatoid arthritis who are at least 50 years old, the live zoster vaccine, if used, should be given before starting DMARDs or biologics whenever possible, ${ }^{8}$ as incidence rates of herpes zoster have been shown to be increased and occur at an earlier age in patients with rheumatic and inflammatory diseases when compared to healthy individuals. ${ }^{6}$ For example, the risk of herpes zoster in rheumatoid arthritis patients in their 40s is approximately equal to or higher than that in healthy older persons in their 60s.

Use of live zoster vaccine has also been shown to be safe and immunogenic when given 2 to 3 weeks before starting tofacitinib in patients with rheumatoid arthritis, but its long-term efficacy was unclear and did not seem to lower the risk of herpes zoster in follow-up of this small cohort. ${ }^{34}$

Due to the disease burden of herpes zoster in this population and uncertainties regardeffective than the live vaccine, but new

recommendations for rheumatology patients are yet to be issued

ing the safety of live zoster vaccine in patients receiving biologic therapies, a randomized, blinded, placebo-controlled trial of live zoster vaccine in patients age 50 and older treated with TNF inhibitors for any on-label or offlabel indication was performed to evaluate for safety and immunogenicity. ${ }^{35}$ The study randomized 617 participants, and there were no cases of disseminated or local varicella infection in the 6 -week period following live zoster vaccination, the at-risk period of concern. The immunologic effectiveness of live zoster vaccine in this trial is still being evaluated.

\section{HEPATITIS B VACCINATION}

In those who were not vaccinated as children, hepatitis $B$ vaccination is not recommended routinely in the United States for adult rheumatic disease patients, but only in those for whom special situations or circumstances increase the risk for transmission. ${ }^{1}$ These circumstances include:

- Hepatitis $\mathrm{C}$ virus co-infection

- Other chronic liver disease

- Human immunodeficiency virus infection

- High-risk sexual behavior

- Injection drug use

- Other high risk for percutaneous or mucosal exposure

- Incarceration

- Travel to countries with high or intermediate endemic hepatitis B.

Practitioners other than rheumatologists may give different recommendations for hepatitis $\mathrm{B}$ vaccination. For example, gastroenterologists routinely recommend it for patients with inflammatory bowel disease regardless of age. ${ }^{48}$

Three hepatitis B vaccines are currently available:

- Heplisav-B, given in a 2-dose series

- Engerix-B or Recombivax HB, given in a 3-dose series

- Twinrix, a combination hepatitis A and B vaccine given in a 3 -dose series.

\section{Effect of DMARDS}

on hepatitis B vaccine effectiveness

The effect of most DMARDs on hepatitis B vaccine immunogenicity has not been evaluated (Table 2); however, TNF inhibitors and ustekinumab have been shown to reduce it. $37,38,39$ Response to the hepatitis $B$ vaccine depends on T-cell activation, and the impairment of T-cell response caused by TNF inhibitors and ustekinumab (and presumably other IL-12/23 inhibitors) is thought to lead to the diminished response. ${ }^{49}$ Several strategies may be needed to improve the immune response to hepatitis $B$ vaccine, including repeated vaccine series, intradermal vaccine administration, development of new vaccine adjuvants, and high-dose vaccines.

A high-dose vaccine containing $40 \mu \mathrm{g} / \mathrm{mL}$ (the usual dose is $20 \mu \mathrm{g} / \mathrm{mL}$ ) was studied in 109 patients with various rheumatologic and inflammatory diseases who were treated with TNF inhibitors or ustekinumab. ${ }^{38}$ The development of a protective antibody titer was seen in $49.3 \%$ of patients who received the standard-dose vaccine and in $61.1 \%$ of those given 
the high-dose vaccine. The difference was not statistically significant, however $(P=.246)$.

Given the likelihood of nonresponse in these groups, it is important that the clinician evaluate for response with postvaccine hepatitis B surface antibody titers to determine if protection has been achieved, with adequate seroprotection typically defined as a titer of 10 $\mathrm{mIU} / \mathrm{mL}$ or higher. ${ }^{50}$

\section{HUMAN PAPILLOMAVIRUS VACCINATION}

Vaccination against HPV is recommended for all adults through age 26, with initial vaccination routinely recommended in adolescents at age 11 or $12 . .^{1,51}$ Using shared decision-making, HPV vaccination may also be offered to those ages 27 to 45 . The age of initial HPV vaccination determines the number of vaccinations given in the series, with a total of 2 or 3 doses comprising a complete series.

Although $3 \mathrm{HPV}$ vaccines are licensed for use, only the 9-valent HPV vaccine (Gardasil 9) is available in the United States; it covers HPV types 6, 11, 16, 18, 31, 33, 45, 52, and 58. Most HPV-associated cancers are caused by HPV types 16 or 18 . There is no recommendation to alter the vaccination schedule for HPV in immunocompromised conditions.

Women with immune-mediated inflammatory diseases and those receiving immunosuppressive medications are at higher risk of HPV infection leading to high-grade cervical dysplasia and cervical cancer. However, vaccination rates are low. ${ }^{7,52}$ Given these concerns, it is important to be aware of barriers to care. Many patients with immune-mediated inflammatory diseases receive vaccinations from their rheumatologists, who may not routinely stock the HPV vaccine. Further, given the complexity of many immune-mediated inflammatory diseases, discussions about preventive care may be deferred. Efforts should be made by both rheumatologists and those in the primary care specialties to encourage vaccination.

\section{Effects of DMARDs} on HPV vaccine effectiveness

Few studies have examined the effects of DMARDs on the immunogenicity of the
HPV vaccine (Table 2). A 2013 prospective, controlled observational study compared the immunogencity of a bivalent HPV vaccine in 68 girls with juvenile idiopathic arthritis compared with 55 healthy girls. ${ }^{40}$ Use of methotrexate did not affect seroconversion. In addition, the rates of seroconversion were not significantly lower in the patients receiving TNF inhibitors; however, the number of patients was considered to be too low to draw strong conclusions.

The effect of TNF inhibitors on HPV vaccine effectiveness was also evaluated in a prospective cohort of 37 female patients ages 9 to 26 with inflammatory bowel disease compared with matched healthy controls from a database. ${ }^{41}$ Patients treated with the TNF inhibitors adalimumab or infliximab comprised $51 \%$ of the cohort, and the remaining $49 \%$ were on other immunomodulators including azathioprine, 6-mercaptopurine, methotrexate, cyclosporine, and tacrolimus. Overall, there was no difference in rates of seropositivity between the inflammatory bowel disease patients and the healthy controls.

There are currently no studies evaluating the effects of abatacept, Janus kinase inhibitors, rituximab, anti-IL-6, anti-IL-17, or antiIL-12/23 inhibitors on the immunogenicity of the HPV vaccine.

\section{TAKE-HOME POINTS}

- Immunocompromised patients are at increased risk of infection due to their primary condition or secondarily due to treatment.

- Vaccination provides an important method of prevention, but use of live vaccines is not recommended in severely immunocompromised persons.

- Non-live vaccines can be used at any time, although preferably they should be given before use of DMARDs in order to minimize negative effects on immunogenicity where they exist.

- For current DMARD users, temporarily holding methotrexate for influenza vaccination could be considered, and most importantly for rituximab, vaccination should occur near the end of the treatment interval 1 month before the next planned dose.

\section{TNF inhibitors and ustekinumab reduce hepatitis B vaccine immunogenicity}




\section{REFERENCES}

1. Freedman $M$, Kroger A, Hunter P, Ault KA; Advisory Committee on Immunization Practices. Recommended adult immunization schedule, United States, 2020. Ann Intern Med 2020; 172(5):337-347. doi:10.7326/M20-0046

2. Rubin LG, Levin MJ, Ljungman $P$, et al. 2013 IDSA clinical practice guideline for vaccination of the immunocompromised host. Clin Infect Dis 2014; 58(3):e44-e100. doi:10.1093/cid/cit684

3. Curtis JR, Xie F, Chen L, et al. The comparative risk of serious infections among rheumatoid arthritis patients starting or switching biological agents. Ann Rheum Dis 2011; 70(8):1401-1406. doi:10.1136/ard.2010.146365

4. Zink A, Manger B, Kaufmann J, et al. Evaluation of the RABBIT Risk Score for serious infections. Ann Rheum Dis 2014; 73(9):1673-1676. doi:10.1136/annrheumdis-2013-203341

5. Curtis JR, Xie F, Chen L, et al. Use of a disease risk score to compare serious infections associated with anti-tumor necrosis factor therapy among high- versus lower-risk rheumatoid arthritis patients. Arthritis Care Res (Hoboken) 2012; 64(10):1480-1489. doi:10.1002/acr.21805

6. Yun H, Yang S, Chen L, et al. Risk of herpes zoster in autoimmune and inflammatory diseases: implications for vaccination. Arthritis Rheumatol 2016; 68(9):2328-2337. doi:10.1002/art.39670

7. Furer V, Rondaan C, Heijstek M, et al. Incidence and prevalence of vaccine preventable infections in adult patients with autoimmune inflammatory rheumatic diseases (AIIRD): a systemic literature review informing the 2019 update of the EULAR recommendations for vaccination in adult patients with AIIRD. RMD Open 2019; 5(2):e001041. doi:10.1136/rmdopen-2019-001041

8. Singh JA, Saag KG, Bridges SL Jr, et al. 2015 American College of Rheumatology guideline for the treatment of rheumatoid arthritis. Arthritis Rheumatol 2016; 68(1):1-26. doi:10.1002/art.39480

9. Cox MM, Patriarca PA, Treanor J. FluBlok, a recombinant hemagglutinin influenza vaccine. Influenza Other Respir Viruses 2008; 2(6):211-219. doi:10.1111/j.1750-2659.2008.00053.x

10. DiazGranados CA, Dunning AJ, Kimmel M, et al. Efficacy of high-dose versus standard-dose influenza vaccine in older adults. N Engl J Med 2014; 371(7):635-645. doi:10.1056/NEJMoa1315727

11. Cowling BJ, Perera RAPM, Valkenburg SA, et al. Comparative Immunogenicity of several enhanced influenza vaccine options for older adults: a randomized, controlled trial. Clin Infect Dis 2019; ciz1034. doi:10.1093/cid/ciz1034

12. Colmegna I, Useche $\mathbf{M}$, Rodriguez $\mathbf{K}$, et al. Efficacy of high-dose versus standard-dose influenza vaccine in seropositive rheumatoid arthritis patients. Arthritis Rheumatol 2018; 70(suppl 10).

13. Sanofi Pasteur Inc. Package Insert: Fluzone high-dose quadrivalent. https://www.fda.gov/media/132238/download. Accessed October 12, 2020.

14. Hua C, Barnetche T, Combe B, Morel J. Effect of methotrexate, anti-tumor necrosis factor a, and rituximab on the immune response to influenza and pneumococcal vaccines in patients with rheumatoid arthritis: a systematic review and meta-analysis. Arthritis Care Res (Hoboken) 2014; 66(7):1016-1026. doi:10.1002/acr.22246

15. Park JK, Lee MA, Lee EY, et al. Effect of methotrexate discontinuation on efficacy of seasonal influenza vaccination in patients with rheumatoid arthritis: a randomised clinical trial. Ann Rheum Dis 2017; 76(9):1559-1565 doi:10.1136/annrheumdis-2017-211128

16. Alten R, Bingham CO 3rd, Cohen SB, et al. Antibody response to pneumococcal and influenza vaccination in patients with rheumatoid arthritis receiving abatacept. BMC Musculoskelet Disord 2016; 17:231.

doi:10.1186/s12891-016-1082-z

17. Winthrop KL, Silverfield J, Racewicz A, et al. The effect of tofacitinib on pneumococcal and influenza vaccine responses in rheumatoid arthritis. Ann Rheum Dis 2016; 75(4):687-695. doi:10.1136/annrheumdis-2014-207191

18. van Assen S, Holvast A, Benne CA, et al. Humoral responses after influenza vaccination are severely reduced in patients with rheumatoid arthritis treated with rituximab. Arthritis Rheum 2010; 62(1):75-81. doi:10.1002/art.25033

19. Bingham CO 3rd, Looney RJ, Deodhar A, et al. Immunization responses in rheumatoid arthritis patients treated with rituximab: results from a controlled clinical trial. Arthritis Rheum 2010; 62(1):64-74. doi:10.1002/art.25034

20. Tsuru T, Terao K, Murakami M, et al. Immune response to influenza vaccine and pneumococcal polysaccharide vaccine under IL-6 signal inhibition therapy with tocilizumab. Mod Rheumatol 2014; 24(3):511-516. doi:10.3109/14397595.2013.843743

21. Furer V, Zisman D, Kaufman I, et al. Immunogenicity and safety of vaccination against seasonal influenza vaccine in patients with psoriatic arthritis treated with secukinumab. Vaccine 2020; 38(4):847-851. doi:10.1016/j.vaccine.2019.10.081

22. Chioato A, Noseda E, Stevens M, Gaitatzis N, Kleinschmidt A, Picaud H. Treatment with the interleukin-17Ablocking antibody secukinumab does not interfere with the efficacy of influenza and meningococcal vaccinations in healthy subjects: results of an open-label, parallelgroup, randomized single-center study. Clin Vaccine Immunol 2012; 19(10):1597-1602. doi:10.1128/CVI.00386-12

23. Richi P, Martín MD, de Ory F, et al. Secukinumab does not impair the immunogenic response to the influenza vaccine in patients. RMD Open 2019; 5(2):e001018. doi:10.1136/rmdopen-2019-001018

24. Kapetanovic MC, Saxne T, Sjöholm A, Truedsson L, Jönsson G, Geborek P. Influence of methotrexate, TNF blockers and prednisolone on antibody responses to pneumococcal polysaccharide vaccine in patients with rheumatoid arthritis. Rheumatology (Oxford) 2006 45(1):106-111. doi:10.1093/rheumatology/kei193

25. Nazi I, Kelton JG, Larché M, et al. The effect of rituximab on vaccine responses in patients with immune thrombocytopenia. Blood 2013; 122(11):1946-1953. doi:10.1182/ blood-2013-04-494096

26. Gomez EV, Bishop JL, Jackson K, Muram TM, Phillips D. Response to tetanus and pneumococcal vaccination following administration of ixekizumab in healthy participants. BioDrugs 2017; 31(6):545-554. doi:10.1007/s40259-017-0249-y

27. Brodmerkel C, Wadman E, Langley RG, et al. Immune response to pneumococcus and tetanus toxoid in patients with moderate-to-severe psoriasis following long-term ustekinumab use. J Drugs Dermatol 2013; 12(10):11221129. pmid:24085047

28. Kapetanovic MC, Roseman C, Jönsson G, Truedsson L, Saxne T, Geborek P. Antibody response is reduced following vaccination with 7-valent conjugate pneumococcal vaccine in adult methotrexate-treated patients with established arthritis, but not those treated with tumor necrosis factor inhibitors. Arthritis Rheum 2011 63(12):3723-3732. doi:10.1002/art.30580

29. Farmaki E, Kanakoudi-Tsakalidou F, Spoulou V, et al. The effect of anti-TNF treatment on the immunogenicity and safety of the 7-valent conjugate pneumococcal vaccine in children with juvenile idiopathic arthritis. Vaccine 2010; 
28(31):5109-5113. doi:10.1016/j.vaccine.2010.03.080

30. Crnkic Kapetanovic M, Saxne T, Jönsson G, Truedsson L, Geborek P. Rituximab and abatacept but not tocilizumab impair antibody response to pneumococcal conjugate vaccine in patients with rheumatoid arthritis. Arthritis Res Ther 2013; 15(5):R171. doi:10.1186/ar4358

31. Winthrop KL, Bingham CO 3rd, Komocsar WJ, et al. Evaluation of pneumococcal and tetanus vaccine responses in patients with rheumatoid arthritis receiving baricitinib: results from a long-term extension trial substudy. Arthritis Res Ther 2019; 21(1):102. doi:10.1186/s13075-019-1883-1

32. Winthrop KL, Korman N, Abramovits W, et al. T-cellmediated immune response to pneumococcal conjugate vaccine (PCV-13) and tetanus toxoid vaccine in patients with moderate-to-severe psoriasis during tofacitinib treatment. J Am Acad Dermatol 2018; 78(6):1149-1155. e1. doi:10.1016/j.jaad.2017.09.076

33. Kapetanovic MC, Roseman C, Jonsson G, Truedsson L, Saxne T, Geborek P. Rituximab and methotrexate but not TNF-blockers are associated with impaired antibody response following pneumococcal vaccination using 7-valent conjugate vaccine (Prevenar) in patients with established rheumatoid arthritis. Arthritis Rheum 2011. https://acr.confex.com/acr/2011/webprogram/Paper19167.html. Accessed October 12, 2020.

34. Winthrop KL, Wouters AG, Choy EH, et al. The safety and immunogenicity of live zoster vaccination in patients with rheumatoid arthritis before starting tofacitinib: a randomized phase ii trial. Arthritis Rheumatol 2017; 69(10):1969-1977. doi:10.1002/art.40187

35. Curtis J, Bridges S, Cofield S, et al. Results from a randomized controlled trial of the safety of the live varicella vaccine in TNF-treated patients. Arthritis Rheumatol 2019; 71 (suppl 10). Accessed October 12, 2020.

36. Parrino J, McNeil SA, Lawrence SJ, et al. Safety and immunogenicity of inactivated varicella-zoster virus vaccine in adults with hematologic malignancies receiving treatment with anti-CD20 monoclonal antibodies. Vaccine 2017; 35(14):1764-1769. doi:10.1016/j.vaccine.2016.10.055

37. Gisbert JP, Villagrasa JR, Rodríguez-Nogueiras A, Chaparro $\mathbf{M}$. Efficacy of hepatitis $B$ vaccination and revaccination and factors impacting on response in patients with inflammatory bowel disease. Am J Gastroenterol 2012; 107(10):1460-1466. doi:10.1038/ajg.2012.79

38. Haykir Solay A, Eser F. High dose hepatitis B vaccine is not effective in patients using immunomodulatory drugs: a pilot study. Hum Vaccin Immunother 2019; 15(5):1177-1182. doi:10.1080/21645515.2019.1574151

39. Salinas GF, De Rycke L, Barendregt B, et al. Anti-TNF treatment blocks the induction of T cell-dependent humoral responses. Ann Rheum Dis 2013; 72(6):1037-1043. doi:10.1136/annrheumdis-2011-201270

40. Heijstek MW, Scherpenisse M, Groot N, et al. Immunogenicity and safety of the bivalent HPV vaccine in female patients with juvenile idiopathic arthritis: a prospective controlled observational cohort study. Ann Rheum Dis 2014; 73(8):1500-1507.

doi:10.1136/annrheumdis-2013-203429
41. Jacobson DL, Bousvaros A, Ashworth L, et al. Immunogenicity and tolerability to human papillomaviruslike particle vaccine in girls and young women with inflammatory bowel disease. Inflamm Bowel Dis 2013; 19(7):1441-1449. doi:10.1097/MIB.0b013e318281341b

42. Nakafero G, Grainge MJ, Myles PR, et al. Effectiveness of inactivated influenza vaccine in autoimmune rheumatic diseases treated with disease-modifying anti-rheumatic drugs. Rheumatology (Oxford) 2020. [Epub ahead of print] doi:10.1093/rheumatology/keaa078

43. Dooling KL, Guo A, Patel M, et al. Recommendations of the Advisory Committee on Immunization Practices for Use of Herpes Zoster Vaccines. MMWR Morb Mortal Wkly Rep 2018; 67(3):103-108. doi:10.15585/mmwr.mm6703a5

44. MerckVaccines. Supply status. https://ordering.merckvaccines.com/supply-status. Accessed October 12, 2020.

45. Lal H, Cunningham AL, Godeaux $O$, et al. Efficacy of an adjuvanted herpes zoster subunit vaccine in older adults. N Engl J Med 2015; 372(22):2087-2096 doi:10.1056/NEJMoa1501184

46. Stevens E, Weinblatt ME, Massarotti E, Griffin F, Desai S. FRI0068 Safety of the zoster recombinant adjuvanted vaccine in rheumatoid arthritis patients: a single center's experience with 300 patients. Ann Rheum Dis 2019; 78(suppl 2):695.

47. Centers for Disease Control and Prevention. Vaccine recommendation and guidelines of the ACIP. Contraindications and precautions. https://www.cdc.gov/vaccines/hcp/ acip-recs/general-recs/contraindications.html. Accessed October 12, 2020.

48. Terrault NA, Lok ASF, McMahon BJ, et al. Update on prevention, diagnosis, and treatment of chronic hepatitis B: AASLD 2018 hepatitis B guidance. Hepatology 2018; 67(4):1560-1599. doi:10.1002/hep.29800

49. Saco TV, Strauss AT, Ledford DK. Hepatitis B vaccine nonresponders: possible mechanisms and solutions. Ann Allergy Asthma Immunol 2018; 121(3):320-327. doi:10.1016/j.anai.2018.03.017

50. Schillie S, Vellozzi C, Reingold A, et al. Prevention of hepatitis B virus infection in the United States: recommendations of the Advisory Committee on Immunization Practices. MMWR Recomm Rep 2018; 67(1):1-31. doi:10.15585/mmwr.rr6701a1

51. Meites E, Szilagyi PG, Chesson HW, Unger ER, Romero JR, Markowitz LE. Human papillomavirus vaccination for adults: updated recommendations of the Advisory Committee on Immunization Practices. MMWR Morb Mortal Wkly Rep 2019; 68(32):698-702 doi:10.15585/mmwr.mm6832a3

52. Feldman $\mathrm{CH}$, Kim SC. Should we target patients with autoimmune diseases for human papillomavirus vaccine uptake?. Expert Rev Vaccines 2014; 13(8):931-934. doi:10.1586/14760584.2014.930346

Address: Jeffrey R. Curtis, MD, MS, MPH, Division of Clinical Immunology and Rheumatology, University of Alabama at Birmingham, 1720 2nd Avenue South, FOT 839, Birmingham, AL 35294; jrcurtis@uabmc.edu 